\title{
Chronic sputum production: correlations between clinical features and findings on high resolution computed tomographic scanning of the chest
}

\author{
Ian E Smith, Erik Jurriaans, Stefan Diederich, Nabeel Ali, John M Shneerson, Christopher \\ D R Flower
}

\begin{abstract}
Background - There are few published data on the correlation between the clinical findings in subjects with chronic sputum production and the appearances on high resolution computed tomographic (HRCT) scans of the chest.

Methods - HRCT scanning of the chest was performed on 40 subjects with chronic sputum production. Three readers independently reported the scans for the presence or absence of bronchiectasis and the extent of bronchiectasis on the basis of the percentage of involved bronchi in each lobe. Relationships were sought between these findings and the clinical history, physical examination, and laboratory investigations.
\end{abstract}

Results - HRCT scanning showed that 27 subjects had bronchiectasis. Of the clinical features only the continual production of purulent sputum and childhood pertussis were associated with bronchiectasis. There was a positive correlation between the extent of bronchiectasis and dyspnoea, and a negative correlation with forced expiratory volume in one second but not with forced vital capacity.

Conclusions - These results indicate that, in subjects with chronic sputum production, only a few clinical features show any correlation with the presence or extent of bronchiectasis as visualised on HRCT scans.

(Thorax 1996;51:914-918)

Keywords: bronchi, abnormalities, bronchiectasis, high resolution computed tomography (HRCT).

Chronic sputum production is a frequent complaint of patients who present to a chest clinic, and chronic bronchitis or bronchiectasis is often a suspected cause. Bronchography was formerly considered to be the best method of diagnosing bronchiectasis, and the relationships between the bronchographic appearances and the clinical features in subjects who produce chronic sputum have been reported. ${ }^{1}$ However, bronchography is now rarely performed, having been largely superseded by high resolution computed tomographic (HRCT) scanning of the chest.
Criteria for the diagnosis of bronchiectasis on CT scanning were first established by Naidich in $1982^{2}$ and elaborated in subsequent studies using HRCT scanning. ${ }^{3}$ Using these diagnostic criteria HRCT scanning has been shown to be comparable in sensitivity and specificity to bronchography. ${ }^{34}$ However, there are few reported data on the associations between the diagnosis of bronchiectasis by HRCT scanning and the clinical features of the disease. We have therefore carried out a prospective study of a group of patients who presented with chronic sputum production to examine the associations between the clinical and laboratory findings, and the diagnosis of bronchiectasis on HRCT scanning.

\section{SUBJECTS}

During a six month period all patients presenting to a respiratory outpatient department for investigation of sputum production which had persisted for more than three months were interviewed. In order to exclude patients with possible reversible bronchiectasis, those who described an exacerbation of their symptoms that is, an increase in sputum production, fever or worsening dyspnoea in the six weeks prior to the interview - were excluded. Forty patients (16 men) were recruited. The study was approved by the local ethics committee and all patients gave their informed consent.

\section{Methods}

CLINICAL EVALUATION

All patients were assessed by one of two physicians and a standard proforma was completed which recorded the duration of cough and of sputum production. They were asked to estimate daily sputum volume by comparison with a teaspoon, tablespoon, an egg cup full, or a teacup full, which were taken as $5,10,50$, and $150 \mathrm{ml}$, respectively. The number of exacerbations per year (that is, increase in sputum production, dyspnoea, fever), production of purulent sputum (graded as never, only during exacerbations, or continual), a history of haemoptysis (graded as never, not for five years, only with exacerbations, or at any time) and dyspnoea (graded as absent, on climbing stairs, on the flat, or on minimal exertion) were also recorded. A past history of childhood respiratory disease was sought from the patient and details of smoking habits were recorded. A general physical examination was performed 
with particular attention to the presence and site of crackles. None of the patients had had chest physiotherapy prior to the examination.

\section{LABORATORY INVESTIGATIONS}

Blood samples were taken for measurement of the full blood count, erythrocyte sedimentation rate (ESR), Aspergillus precipitin titre, and immunoglobulin levels. None of the patients had other features suggestive of cystic fibrosis so no genetic analyses were performed. Sputum samples were sent for microscopic examination and culture. Forced expiratory volume in one second $\left(\mathrm{FEV}_{1}\right)$ and forced vital capacity (FVC) were measured using a dry spirometer (Vitalograph Ltd). The best of three attempts was recorded and expressed as the percentage of the predicted values. ${ }^{5}$

\section{RADIOLOGY}

All patients were scanned using a Somatom Plus Scanner (Siemens Corporation, New Jersey, USA). Sections $1 \mathrm{~mm}$ thick were obtained in full inspiration at $10 \mathrm{~mm}$ intervals from the level of the clavicles to the lung bases. Where indicated, additional sections were obtained in expiration, in the prone position, or with cephalad angulation. Images were reconstructed using a high resolution algorithm. All HRCT examinations were assessed using hard copy with a window width of 1800 and centred at -850 Hounsfield units. The HRCT images were reviewed independently by three radiologists. In cases where there was disagreement the majority view was taken. The HRCT images were analysed to determine whether bronchiectasis was present. ${ }^{23}$ Bronchiectasis $^{3}$ was diagnosed when a bronchus had a diameter greater than the accompanying pulmonary artery and failed to taper either when viewed in the plane of the scan or on consecutive slices.

Bronchiectasis was defined as cylindrical when the bronchial lumen had a smooth tubular outline, as varicose when the lumen had a variable diameter producing a beaded appearance, and as cystic when the dilated bronchi had the appearance of rings or clusters of cysts. For the purposes of this study bronchial dilatation was considered to be present if the average internal diameter of the bronchus was greater than $110 \%$ of the diameter of the accompanying artery with the diameters measured using a calliper. This is a quantitative development of a previously described method. ${ }^{3}$ Note was also made of the presence of bronchi visible in the peripheral third of the lung field.

When bronchiectasis was diagnosed an estimate of its extent was made as follows. The percentage of involved bronchi in each lobe was calculated and graded as 0 if there was no evidence of bronchiectasis, 1 if less than $25 \%$ of the bronchi were abnormal, 2 if $25-49 \%$ of the bronchi were abnormal, 3 if $50-74 \%$ of the bronchi were abnormal, and 4 if $75 \%$ or more of the bronchi were abnormal. The lingula was counted as a separate lobe and the maximum possible score for the extent of bronchiectasis was therefore 24. This is an adaptation of methods previously described which have scored the extent of bronchiectasis on the basis of the number of involved segments. ${ }^{6} 7$ These methods are more time consuming and the individual segments cannot always be reliably identified.

Non-bronchial abnormalities, if present, were described in terms of volume loss, consolidation, emphysema, pulmonary nodules or masses, diffuse pulmonary infiltrate, and pleural fluid or thickening.

\section{STATISTICAL ANALYSIS}

Excluding age, FVC, and white cell coun (WCC), the data were not normally distributed and so non-parametric tests were used for most of the analyses. We used the SPSS package (SPSS Inc, Chicago, USA) and results of $p$ $<0.05$ were considered significant.

Interobserver variability in the diagnosis of bronchiectasis was examined using the kappa statistic. Differences were sought between patients with and without bronchiectasis on HRCT scanning. The groups were compared by the $\chi^{2}$ test, using Yates' continuity correction, for the presence or absence of continual purulent sputum, and by Fisher's exact test for the presence or absence of rhinitis, dyspnoea grade $>2$, haemoptysis, a history of smoking, and a positive sputum culture. Comparisons were made using the Wilcoxon rank sum test with respect to age, length of history, sputum volume, cigarette pack years, lung function, and WCC. In the group with bronchiectasis correlation between the extent of bronchiectasis and age, length of history, sputum volume, cigarette pack years, lung function, and WCC was sought using Kendall's rank correlation coefficient.

A significant correlation was demonstrated between the extent of bronchiectasis and the $\mathrm{FEV}_{1}$. The factors affecting $\mathrm{FEV}_{1}$ were further investigated using stepwise linear regression with extent, presence of bronchiolitis, sputum volume, cigarette pack years, and age as independent variables.

\section{Results}

Complete data from the clinical history and examination were available for all 40 patients except for the record of cough and sputum years for one patient and the frequency of exacerbations in another. The full blood count was available for 35 patients, ESR for 30, Aspergillus precipitins for 24, immunoglobulins for 31 , sputum microbiology for 29 , and spirometric results for 35 patients.

CLINICAL AND LABORATORY DATA

The mean (SD) age of the 40 patients was 56 (16.03) years (range 19-81). Twenty six had a history of childhood respiratory disease with six suffering recurrent bronchitis, three asthma, nine pneumonia, five pertussis alone, one measles alone, one pertussis and measles, and one pertussis and tuberculosis. Two patients had hypogammaglobulinaemia, both of whom were on replacement therapy. Rhinitis was a complaint in 14 patients. The median duration of cough was 12 years (range 1-50) and of sputum production was 10 years (range 
Table 1 Frequency of individual bronchial abnormalities on HRCT scanning in patients with and without bronchiectasis

\begin{tabular}{lll}
\hline & $\begin{array}{l}\text { Bronchiectasis on } \\
\text { HRCT }(n=27)\end{array}$ & $\begin{array}{l}\text { No bronchiectasis } \\
\text { on HRCT }(n=13)\end{array}$ \\
\hline Failure of bronchi to taper & 27 & 0 \\
Bronchial dilatation & 27 & 0 \\
Bronchi seen in the peripheral third of the lung field & 22 & 3 \\
Abnormal bronchial contents & 8 & 1 \\
\hline
\end{tabular}

Table 2 Clinical findings

\begin{tabular}{|c|c|c|}
\hline & $\begin{array}{l}\text { Bronchiectasis on } \\
\text { HRCT }(n=27)\end{array}$ & $\begin{array}{l}\text { No bronchiectasis on } \\
\operatorname{HRCT}(n=13)\end{array}$ \\
\hline Mean (SD) age (years) & $58.9(13.96)$ & $53.2(22.44)$ \\
\hline Non-smokers & $18(67 \%)$ & $7(54 \%)$ \\
\hline Pack years ${ }^{\star \star}$ & 17 (range 5-40) & 30 (range $5-50$ ) \\
\hline \multicolumn{3}{|l|}{ Childhood respiratory disease } \\
\hline None & 9 & 5 \\
\hline Bronchitis & 3 & 3 \\
\hline Asthma & 1 & 2 \\
\hline Pneumonia & 6 & 3 \\
\hline Pertussis & 7 & 0 \\
\hline Measles & 2 (one with pertussis) & 0 \\
\hline Tuberculosis & 1 (also had pertussis) & 0 \\
\hline Rhinitis & $18(66.6 \%)$ & $5(38.5 \%)$ \\
\hline Sputum years ${ }^{\star}$ & 13 (range $1-50$ ) & 5 (range $0.5-15$ ) \\
\hline Sputum volume ${ }^{\star}$ (ml per day) & 25 (range $10-200)$ & 23 (range $10-100$ ) \\
\hline Exacerbations ${ }^{\star}$ (per year) & 3 (range $1-10)$ & 4 (range $1-5$ ) \\
\hline \multicolumn{3}{|l|}{ Purulent sputum } \\
\hline Never & $1(3.7 \%)$ & 0 \\
\hline Only in exacerbations & $11(40.7 \%)$ & $11(84.6 \%)$ \\
\hline Continual & $15(55.6 \%)$ & $2(15.4 \%)$ \\
\hline \multicolumn{3}{|l|}{ Dyspnoea score } \\
\hline$>1$ & $16(60 \%)$ & $10(77 \%)$ \\
\hline$>2$ & $6(22 \%)$ & $3(23 \%)$ \\
\hline
\end{tabular}

* Median; ${ }^{\star \star}$ Median for smokers only.

1-50). Using the Wilcoxon ranked pairs test the duration of these two symptoms was not significantly different. The median number of exacerbations per year was four. One patient had never had purulent sputum, 22 only had it during exacerbations, and 17 always had purulent sputum.

Twenty three patients had never had haemoptysis, three not for five years, 12 only during exacerbations, and only two had experienced haemoptysis in the last five years not associated with an exacerbation. Fourteen patients had no dyspnoea, 17 were dyspnoeic on climbing stairs, eight while walking on the flat, and one on minimal exertion. Only two patients were current smokers, 13 were ex-smokers, and 25 were non-smokers. The median number of pack years for those who had ever smoked was 25.

On examination none of the patients was clubbed or cyanosed, or had peripheral oedema, a raised JVP or a right ventricular heave. Chest crackles were present in 15 patients.

The mean WCC was $8.75(2.3) \times 10^{\%} / 1$ with five patients having a value of more than 11 . The mean ESR was $17.5 \mathrm{~mm}$ in the first hour and 12 patients had a value of more than 20 . Aspergillus precipitins were absent in all patients tested. No new diagnoses of hypogammaglobulinaemia were made. Sputum culture revealed no growth in 18 patients, Haemophilus influenzae in eight, Pneumococcus in one, and Pseudomonas aeruginosa in two.

HRCT FINDINGS

Of the 40 patients, $27(67.5 \%)$ were found to have bronchiectasis of whom $18(66.7 \%)$ were women. It was cylindrical in 16, varicose in nine, and cystic in two. Neither of the patients with hypogammaglobulinaemia had bronchiectasis. The frequency of bronchial abnormalities is shown in table 1. Patients with bronchiectasis were more likely to have fluid filled bronchi $(p<0.005)$ and to have bronchi visible in the peripheral third of the lung ( $p$ $<0.005$ ). Agreement between the three readers on the diagnosis of bronchiectasis was good $($ kappa $=0.76)$.

Five patients had centrilobular tubular branching opacities in some secondary lobules in keeping with an inflammatory bronchiolitis. Nine patients had focal areas of reduced parenchymal attenuation adjacent to bronchiectatic bronchi suggestive of an associated obliterative bronchiolitis. No patient had consolidation, a diffuse pulmonary infiltrate or any pleural abnormality.

RELATIONSHIP BETWEEN THE PRESENCE OF BRONCHIECTASIS AND CLINICAL AND LABORATORY FINDINGS

The clinical findings are summarised for patients with and without bronchiectasis in table 2. Patients with bronchiectasis were more likely to produce purulent sputum continually than those with no evidence of bronchiectasis ( $p<0.05)$. No significant differences were found between the two groups for age, duration of cough, duration of sputum production, sputum volume, frequency of exacerbations, dyspnoea, or smoking pack years. There was no association between bronchiectasis and haemoptysis whether this was within the last five years or at any time previously. However, both patients with haemoptysis not associated with exacerbations had bronchiectasis. HRCT evidence for obliterative bronchiolitis was associated with a positive smoking history ( $p$ $<0.01$ ).

All seven patients who had suffered childhood pertussis had bronchiectasis, a significant association using Fisher's exact test ( $p$ $<0.05)$. In this subgroup the patients were not significantly older than the other patients but did have a significantly longer duration of symptoms (Wilcoxon rank sum test $p=0.02$ for cough duration), suggesting a causal link between their childhood disease and subsequent bronchiectasis. There was no such relationship for other childhood diseases. No other significant differences were found between the groups with and without bronchiectasis.

The concordance between the presence of crackles on auscultation and the location of bronchiectasis on the HRCT scan was calculated for each lung and the results are given in table 3.

Using the $\chi^{2}$ test there was no association between the presence of crackles and bronchiectasis on a per lung basis. The laboratory findings are given in table 4 . No statistically significant differences were seen between patients with and without bronchiectasis.

In the group with no bronchiectasis on the HRCT scan the seven non-smokers had a mean age of 40 (20) years which was 
Table 3 Concordance between clinical examination and bronchiectasis on HRCT scans

\begin{tabular}{lll}
\hline & $\begin{array}{l}\text { Bronchiectasis } \\
\text { on HRCT }\end{array}$ & $\begin{array}{l}\text { No } \\
\text { bronchiectasis } \\
\text { on HRCT }\end{array}$ \\
\hline $\begin{array}{llr}\text { No crackles on auscultation } \\
\text { Crackles on auscultation }\end{array}$ & $\begin{array}{l}30(37.5 \%) \\
13(16.25 \%)\end{array}$ & $\begin{array}{l}30(37.5 \%) \\
7(8.75 \%)\end{array}$ \\
\hline
\end{tabular}

significantly younger than the smokers (mean difference 18.7 years, $p=0.01$ ). Two had hypogammaglobulinaemia which might explain their symptoms, but in the others no cause for their chronic sputum production was apparent.

\section{CORRELATION BETWEEN THE EXTENT OF} BRONCHIECTASIS AND CLINICAL AND LABORATORY FINDINGS

The score for the extent of bronchiectasis ranged from 1 to 19 (median $=5$ ). Within the group with bronchiectasis the only significant correlation between symptoms and the extent of bronchiectasis was for dyspnoea (Kendall's rank correlation coefficient $=0.38, \mathrm{p}=0.01$ ). Using the $\chi^{2}$ test an extent score for bronchiectasis of 8 (equivalent to the involvement of two complete lobes) or more was associated with dyspnoea of grade 1 or more ( $p<0.05$ ). A significant negative correlation was found between the extent of bronchiectasis and $\mathrm{FEV}_{1} \%$ (coefficient $=-0.43, \mathrm{p}<0.01$ ) but not with FVC\%. In the linear regression analysis with $\mathrm{FEV}_{1}$ as the dependent variable only age (sig $\mathrm{T}<0.01$ ) and extent of bronchiectasis (sig $\mathrm{T}=0.01$ ) had a predictive value. The duration of symptoms, number of pack years, and the presence of bronchiolitis were excluded from the equation which took the form :

$$
\begin{aligned}
& \mathrm{FEV}_{1}=4.13+(\text { Age } \times-0.026)+(\text { Extent } \\
& \quad \times-0.088) \\
& \mathrm{R}^{2} \text { was } 0.46 .
\end{aligned}
$$

\section{Discussion}

Patients with chronic sputum production presenting to a respiratory clinic are most frequently diagnosed as having either chronic bronchitis or bronchiectasis. The chest radiograph is rarely diagnostic of bronchiectasis and bronchography is now rarely performed. HRCT scanning has emerged as the investigation of choice in the radiological investigation of possible bronchiectasis. Criteria for the diagnosis of bronchiectasis on CT scanning as first described by Naidich et al were devised in accordance with Reid's pathological classification. ${ }^{9}$ There have been several papers comparing the sensitivity and specificity of CT scanning $^{10-12}$ and, latterly, HRCT scanning ${ }^{43}$ with bronchography in the diagnosis of bronchiectasis, and a sensitivity of $84-97 \%$ has been reported for HRCT scanning. However, little work has been done on the associations between the clinical and laboratory features of bronchiectasis and the HRCT appearances.

Our findings suggest that there is a poor correlation between the CT findings of bronchiectasis and the clinical history and examination. Almost one third of patients with symptoms suggesting a diagnosis of bronchiectasis had no evidence for this on the HRCT scan. This proportion is similar to that found by Currie et al in a study of the bronchographic appearances of 27 patients with chronic sputum production. Eight of their patients had no bronchographic features of bronchiectasis and there were no significant clinical differences between this group and the remaining 19 patients with bronchograms which were diagnostic of bronchiectasis. In our series only the continual production of purulent sputum was associated with the presence of bronchiectasis $(p<0.05)$. We also found the physical examination unreliable in distinguishing patients with and without bronchiectasis. It has been claimed that crackles are a universal finding in bronchiectasis ${ }^{14}$ but in our patients this was not the case and, when crackles were heard, there was poor concordance between their location and the distribution of bronchiectasis on the HRCT scan.

In most of the reports of the CT signs of bronchiectasis no attempt has been made to weight the individual signs or to describe their specificity. Lynch et $a l^{15}$ have cautioned against making the diagnosis of bronchiectasis on the basis of bronchial dilatation alone and emphasised the importance of a lack of bronchial tapering on HRCT scans. In a study comparing HRCT scanning with post resection pathological examination of bronchiectatic lung specimens Kang et $a l^{16}$ found that the CT appearance of abnormal bronchial tapering was $79 \%$ sensitive while bronchial dilatation alone was only $60 \%$ sensitive. In our study bronchiectasis was only diagnosed if bronchial dilatation was associated with a lack of bronchial tapering.

We have described the extent of bronchiectasis on the basis of the percentage of bronchi involved on a lobar basis. This method has been validated against pathological examination of lung specimens resected for bronchiectasis with an overall sensitivity of $87 \% .^{16}$ Previous scoring systems were based on the presence or absence of abnormal bronchi within each segment, with $^{6}$ or without ${ }^{7}$ a weighting factor for the degree of bronchial dilatation. Our system is easier to use and does not appear to be less sensitive as the significant negative correlation between extent of bronchiectasis and the $\mathrm{FEV}_{1} \%$ described in a previ-

Table 4 Laboratory findings

\begin{tabular}{lll}
\hline & Bronchiectasis on HRCT $(n=27)$ & No bronchiectasis on HRCT $(n=13)$ \\
\hline Mean (SD) FEV 1 (\% predicted) & $67.2(26.59)$ & $78.4(24.71)$ \\
Mean (SD) FVC (\% predicted) & $79.3(22.67)$ & $86.7(20.17)$ \\
Positive sputum culture & $9(50 \%)($ missing data for 9 patients) & $2(18.4 \%)$ (missing data for 2 patients) \\
Median erythrocyte sedimentation rate $(\mathrm{mm} / \mathrm{h})$ & $15($ range 5-64) & 19.5 (range 12-38) \\
Mean (SD) white cell count $\left(\times 10^{9} / \mathrm{l}\right)$ & $8.7(2.6)$ & $8.9(1.52)$ \\
\hline
\end{tabular}


ous study $^{7}$ is reproduced with an identical coefficient for Kendall's rank correlation $(-0.43)$. In addition, we have shown a positive correlation between the extent of bronchiectasis and the symptom of dyspnoea. The presence of airflow obstruction in bronchiectasis is well described ${ }^{17-19}$ and is related to prognosis. $^{20}$

In this study of a group of 40 patients with chronic sputum production the only feature of the history, clinical examination, and laboratory investigations which had predictive value for the finding of bronchiectasis on HRCT examination of the chest was the continual production of purulent sputum. In a proportion of patients with symptoms strongly suggestive of bronchiectasis there was no evidence for it on the HRCT scan. In their study of chronic sputum producers Currie et $a l^{1}$ concluded that bronchography can identify the macroscopic anatomical abnormalities of the airways, but medical management should be based on the patient's clinical presentation as active inflammation may not be confined to radiographically abnormal bronchi and some bronchiectatic bronchi may be quiescent. We conclude that, despite technical advances in HRCT scanning, the same limitations remain for the radiographic investigation of chronic sputum production. It is possible that HRCT scanning has a role in following the progression or stability of bronchiectasis with different treatments, but further work is required to demonstrate this.

1 Currie DC, Cooke JC, Morgan AD, Kerr IH, Delany D, Strickland $\mathrm{B}$, et al. Interpretation of bronchograms and chest radiographs in patients with chronic sputum production. Thorax 1987;42:278-84.

2 Naidich DP, McCauley DI, Khouri NF, Stitik FP Siegleman SS. Computed tomography of bronchiectasis. Comput Assist Tomogr 1982;6:437-44.
3 Grenier P, Maurice F, Musset D, Menu Y, Nahun H. Bronchiectasis: assessment by thin-section CT. Radiology 1986 ; 161:95-9.

4 Munro NC, Cooke JC, Currie DC, Strickland B, Cole PJ. Comparison of thin section computed tomography with bronchography for identifying bronchiectatic segments in patients with chronic sputum production. Thorax 1990 45:135-9.

5 Cotes J. Lung function., Oxford: Blackwell Scientific Publications, 1979:329-87.

6 Bhalla M, Turcios N, Aponte V, Jenkins M, Leitman BS, et al. Cystic fibrosis: scoring system with thin section CT. Radiology 1991;179:783-8.

7 Wong-You-Cheong JJ, Leahy BC, Taylor PM, Church SE Airways obstruction and bronchiectasis: correlation with duration of symptoms and extent of bronchiectasis on computed tomography. Clin Radiol 1992;45:256-9.

8 Grenier P, Lenoir S, Beigelman C. Diagnosis of bronchiectasis. Imaging 1992;4:39-48.

9 Reid LM. Reduction in bronchial subdivision in bronchiectasis. Thorax 1950;5:233-47.

10 Silverman PM, Godwin JD. CT/bronchographic correlations in bronchiectasis. $\mathcal{f}$ Comput Assist Tomogr 1987 11:52-6.

11 Cooke JC, Currie DC, Morgan AD, Kerr IH, Delaney D, Strickland B, et al. Role of computed tomography in diagnosis of bronchiectasis. Thorax 1987;42:272-7.

12 Phillips MS, Williams MP, Flower CDR. How useful is computed tomography in the diagnosis and assessment of bronchiectasis? Clin Radiol 1986;37:321-5.

13 Joharjy IA, Bashi SA, Abdullah AK. Value of medium thickness CT in the diagnosis of bronchiectasis. AfR 1987; 149:1133-7.

14 Stanford W, Galvin J. The diagnosis of bronchiectasis. Clin Chest Med 1988;9:691-9.

15 Lynch DA, Newell JD, Tschomper BA, Cink TM, Newman LS, Bethel R. Uncomplicated asthma in adults: comparison of CT appearances of the lungs in asthmatics and healthy subjects. Radiology 1993;188:829-33.

16 Kang KY, Miller RR, Muller N. Bronchiectasis: comparison of pre-operative thin-section CT and pathologic findings in resected specimens. Radiology 1995;195:649-54.

17 Ip M, Lauder JJ, Wong WY, Lam WK, So SY. Multivariate analysis of factors affecting pulmonary function in bronchiectasis. Respiration 1993;60:45-50.

18 Pande JN, Jain BP, Gupta RG, Guleria JS. Pulmonary ventilation and gas exchange in bronchiectasis. Thorax 1971;26:727-33.

19 Cherniak M, Carton RW. Factors associated with respiratory insufficiency in bronchiectasis. Am f Med 1966; 41:562-72.

20 Ellis DA, Thornley PE, Wightman AJ, Walker M, Chalmer J. Crofton JW. Present outlook in bronchiectasis: clinical and social study and review of factors influencing prognosis. Thorax 1981;36:659-64. 